\title{
Combustion Optimization for the Prevention of Backfire in a 2-Liter Class Hydrogen Engine
}

\author{
Young Choi ${ }^{1}$, Cheolwoong Park ${ }^{1}$, Yongrae Kim ${ }^{1}$, Uihyung Yi ${ }^{1}$, Hyungjoon Jang ${ }^{1}$, Byeung jun Lim ${ }^{2}$ \\ ${ }^{1}$ Korea Institute of Machinery and Materials (KIMM) \\ 156 Gajeongbook-ro Yuseong-goo, Daejeon, Korea \\ ychoi@kimm.re.kr; cwpark@kimm.re.kr; yrkim@kimm.re.kr; uhyi@kimm.re.kr; jhj1721@kimm.re.kr \\ ${ }^{2}$ Korea Aerospace Research Institute (KARI) \\ 169-84 Gwahak-ro Yuseong-goo, Daejeon, Korea \\ bjlim@kari.re.kr
}

\section{Extended Abstract}

Hydrogen is known to be an efficient energy carrier with high gravimetric energy density. ${ }^{[1]}$ Application of hydrogen as a fuel to power sources has been tried in many different ways. ${ }^{[27]}$ Although hydrogen has been experimented successfully in combustion devices, abnormal combustion such as backfire, pre-ignition and misfire causes deterioration of thermal efficiency and damage to device safety. ${ }^{[8 \sim 10]}$ Therefore, combustion optimization is necessary to maximize thermal efficiency and guarantee stable utilization of hydrogen. ${ }^{[11]}$ Recently, effort to use liquid hydrogen has been challenged as a fuel for an unmanned aerial vehicle (UAV) that can fly for days at high altitude. The aim of this study is to find the optimum condition in combustion to prevent or at least to minimize the occurrence of backfire in medium size hydrogen engine as a power source of an UAV.

In this study, pure hydrogen was adopted to a 2-liter class reciprocating internal combustion engine with 4 cylinders varying engine speed from idling to 3,000rpm. Hydrogen was injected into the intake port maintaining the supply pressure at 8 bar and spark ignited inside the cylinder during compression stroke. Due to the rapid reactivity of hydrogen, the combustion performance of this experimental engine was comparable to that of gasoline engine and even supreme in terms of thermal efficiency at low load operating conditions. However, severe decrease in engine power was witnessed and combustion stability has been worsened with the increase of engine load due to backfire. In addition, decrease in volumetric efficiency caused from the low heating value of hydrogen per unit volume even worsened engine power output at high load operating conditions.

In order to prevent backfire at high load operating condition, three different approaches were applied throughout this experiment. First, lean combustion was used. The range of equivalence ratio was from 0.5 to 0.8 at full load operating condition. Lean combustion lessened the degree of backfire happening through intake. However, torque output was sharply dropped with the decrease of equivalence ratio. This led to the compromised lean limit at the equivalence ratio of around 0.7. Second, the hydrogen injection timing was varied to the optimal point. Too early injection enhanced mixing between fresh air and hydrogen leading high chance of backfire. In contrast, injected hydrogen was not fully flowed into the combustion chamber if injection was too late. Third, opening and closing timing control of both intake and exhaust valve has been carried out. Hot spots from residual gas inside the cylinder act as an ignition source that could initiate backfire. So, residual gas was intendedly reduced by valve timing control. The intake and exhaust valve opening and closing timing was adjusted to minimize valve overlap period. Although the optimum valve opening and closing timing was found at a certain operating condition, relationship between intake and exhaust valve timing was not generalized due to the different mixture distribution. In general, flow pattern inside the cylinder is unpredictable because mixture and temperature distribution are frequently varied by not only intake and exhaust flow but also squish and tumble motion. Above three approaches affected backfire behavior one another. Therefore, combustion optimization should be carefully considered to prevent backfire and to guarantee safe operation of hydrogen engine. 


\section{References}

[1] I. L. Drell and F. E. Belles, "Survey of hydrogen combustion properties," National Advisory Committee for Aeronautics Report 1383, 1958.

[2] M. R. Swain, "Hydrogen-fueled automotive engine experimental testing to provide an initial design-data base," SAE paper $810350,1981$.

[3] J. B. Heywood, Internal combustion engine fundamentals. Mc Graw Hill, 1988.

[4] L. M. Das, "Exhaust emission characterization of hydrogen-operated engine system: Nature of pollutants and their control techniques," Int. J. of Hydrogen Energy, vol. 16, no. 11, pp. 765-775, 1991.

[5] S. Dunn, "Hydrogen futures: towards a sustainable energy system," Int. J. of Hydrogen Energy, vol. 27, pp. 235-264, 2002.

[6] H. Eichlseder, "The potential of hydrogen internal combustion engines in a future mobility scenario," SAE, p. 200301-2267, 2003.

[7] M. Berckmuller, "Potentials of a charged SI-hydrogen engine," SAE p. 2003-01-3210, 2003.

[8] S. Verhelst, R. Sierens and S. Verstraeten, "A critical review of experimental research on hydrogen fueled SI engines," SAE Paper 2006-01-0430, 2006.

[9] C. M. White, "The hydrogen-fueled internal combustion engine: a technical review," Int. J. of Hydrogen Energy, vol. 31, pp. 1292-1305, 2006.

[10] S. Verhelst and T. Wallner, "Hydrogen-fueled internal combustion engines," Prog Energy Combust Sci, vol. 35, no. 6, pp. 490-527, 2009.

[11] G. H. Lim, S. W. Lee, C. W. Park, Y. Choi and C. G. Kim, "Effect of ignition timing retard strategy on NOx reduction in hydrogen-compressed natural gas blend engine with increased compression ratio," Int. J. of Hydrogen Energy, vol. 39, pp. 2399-2408, 2014. 